$\begin{array}{ll}\text { le portiQue } & \text { Le Portique } \\ \text { Revue de philosophie et de sciences humaines }\end{array}$

$20 \mid 2007$

Gilles Deleuze et Félix Guattari : Territoires et devenirs

\title{
Bords à bords : vers une pensée-musique
}

\section{Pascale Criton}

\section{OpenEdition}

\section{Journals}

Édition électronique

URL : http://journals.openedition.org/leportique/1366

DOI : $10.4000 /$ leportique.1366

ISSN : $1777-5280$

\section{Éditeur}

Association "Les Amis du Portique"

Édition imprimée

Date de publication : 15 décembre 2007

ISSN : 1283-8594

\section{Référence électronique}

Pascale Criton, «Bords à bords : vers une pensée-musique », Le Portique [En ligne], 20 | 2007, mis en ligne le 06 novembre 2009, consulté le 26 mars 2021. URL : http://journals.openedition.org/leportique/ 1366 ; DOI : https://doi.org/10.4000/leportique.1366

Ce document a été généré automatiquement le 26 mars 2021.

Tous droits réservés 


\title{
Bords à bords : vers une pensée- musique
}

\author{
Pascale Criton
}

1 Je voudrais évoquer dans ce texte les échanges particuliers que Gilles Deleuze et Félix Guattari ont tissé entre musique, territoires et devenirs. Au cours des années 1970, époque marquée par le structuralisme, Deleuze et Guattari se tournent vers la question d'un rapport nouveau entre l'expression et les dispositifs de la pensée. Leur point de vue s'établit avec le projet d'une hétérogenèse sémiotique transversale et prend appui sur la recherche d'outils de pensée puisés dans tous les domaines de la représentation. Qu'est-ce qui traverse les champs expressifs, du politique au technique, du social au psychique? La linguistique, l'ethnologie, l'éthologie, la sociologie, la psychiatrie, non moins que les mathématiques, l'esthétique, la littérature, la peinture, le cinéma sont convoqués. C'est dans cette perspective que le domaine particulier de la musique est interrogé. La musique peut-elle être sollicitée pour une pensée transversale? Quels sont ses outils, quel est son champ opératoire ? En quoi la musique peut-elle nous aider à penser?

2 Je me réfèrerai ici aux textes qui posent d'emblée, à mon sens, le noyau stratégique d'une rencontre avec le musical : celui des déterminations spatiales et temporelles ainsi que la question des dynamismes exposée par Deleuze dès Différence et répétition. Une pensée des dynamismes, des déterminations et des différences préside à la formation de ce qui deviendra ultérieurement, avec Félix Guattari, la ritournelle, notion qui développera ses implications avec le territoire et les devenirs.

3 Je me placerai aussi du point de vue du témoin que j'ai été, au fil de cette recherche en marche, dans les années 1970, et plus tard. En effet, Deleuze abordait, en cette période de préparation de Mille Plateaux, des questions concernant la notion de continuum et la façon dont l'emploi du chromatisme en musique pourrait être une piste utile à étudier. Alors jeune musicienne, de passage à un séminaire «du mardi », il se trouvait que le chromatisme était déjà mon principal centre d'intérêt musical. Rien ne prédestinait cette rencontre, si ce n'est le hasard et ses fruits imprévisibles. C'est bien cet intérêt " commun » pour le chromatisme qui nous a tout d'abord rapprochés. Invitée à venir 
me joindre à la réflexion ${ }^{1}$, j'ai appris à mesurer, par la suite, la spécificité de l'acte de penser en philosophie : un art de procéder, de bâtir, d'amener la représentation vers de nouveaux termes. Peut-être ai-je, au fil de cet échange réciproque et durable, « chacun sur son bord » comme on dit, acquis quelques repères dans ce domaine - mais je ne suis pas philosophe pour autant.

Je voudrais donc proposer ici quelques pistes qui montrent la façon dont le musical s'est progressivement mis à jouer comme «modèle » capable de relier du sensible, du collectif et du diagrammatique. Bien que la musique n'ait pas fait l'objet d'un livre, comme c'est le cas pour le cinéma, la peinture ou la littérature, celle-ci occupe néanmoins une place privilégiée dans la pensée de Deleuze et Guattari. Vecteur spécialisé d'un processus transversal de variation, la musique est conviée, invitée à se joindre à l'expérience de la pensée dont la philosophie n'entend pas être l'unique spécialiste. Les outils pluriels de Deleuze et Guattari reposent sur l'élaboration d'un champ hétérogène particulièrement sensible aux questions de méthode. La musique at-elle un rôle à jouer dans la conception d'objets polyvoques, transversaux ? Y a-t-il une matière d'échange propice à une capture du modèle musical ? Et inversement, en quoi les outils de Deleuze et Guattari doivent-ils certaines qualités à leur contact avec des champs exogènes aux habitudes de la pensée philosophique? Auquel cas, en quoi et pourquoi ces outils polyvoques, «trans-catégoriels », nous intéressent-ils aujourd'hui ?

\section{Souvenirs. À propos de « méthode »}

Dans le courant de ces années 1970, Deleuze travaillait sur les notions de machines de guerre et d'appareil d'état. Le chromatisme allait côtoyer la machine de guerre, participer aux opérations de consistance, aux agencements de strates et de plans et se voir associé au travail de la déterritorialisation ${ }^{2}$. Si l'on évoque la question de la " méthode », il faudrait tout d'abord entendre un ensemble de précautions préalables, de dispositions et de "modes d'emplois ", loin d'un cadre fixe. La précision que Deleuze apportait, en ce qui concerne les façons de « contracter les relations » était un véritable enseignement. L'organisation de différents régimes d'attention constituait un parcours exemplaire de l'enchaînement des opérations de la pensée - qu'il veillait pédagogiquement à signaler et à nommer, et qui, à certains égards, n'était pas sans relation avec des mouvements de la pensée que nous employons en musique, dans l'analyse musicale et jusque dans la composition. Deleuze accordait une attention toute particulière, par exemple, à la question de l'orientation dans la pensée. Convoquer des lignes indépendantes et créer un état flottant, constituer une nébuleuse, agitée de contractions locales, de petites tensions, par la suite extraites, déplacées, apposées. C'est dans cette phase de coexistence que s'éprouvent les traits différentiels, les couplages et les captures réciproques qui préparent la déterritorialisation. C'est le temps des mises en relation, sans insistance, un équilibre entre le tact et l'esquisse. Laisser l'idée se fabriquer, par strates successives, reprises, abandons, effets retard: « De quoi a-t-on besoin pour que cela tienne? ? L'idée est entre, dans la sélection et le réenchaînement.

6 Cette phase du travail, celle de la recherche de points remarquables, de la spécification, de l'expression de singularités, repose sur un ensemble de préalables qui vaut pour une méthode. L'agent recherché - le différenciant, s'exprime avec la validation des relations, le jeu de déterminations réciproques et l'engendrement de couplages. 
Recherche guettant le mouvement d'un « drama » formé par les concepts et les états de choses eux-mêmes, sous la représentation, sous le logos. Il y aura donc des opérations pour réaliser la mise en place d'un "théâtre des propriétés et événements ». Il y aura des conditions préalables pour situer une consistance et permettre une processualité active: « Un ensemble flou, une synthèse de disparates n'est définie que par un degré de consistance rendant précisément possible la distinction des éléments disparates qui le constituent (discernabilité) ${ }^{3}$.

7 C'est d'ailleurs autour de la notion consistance que la musique apparaît comme le site privilégié d'une hétérogenèse, d'une pratique de "chevauchement» catégoriel. Si la notion de ritournelle est composite, "trans-catégorielle", synthèse de composantes liées au territoire, à l'affect et aux codes, elle repose sur les tenants d'une esthétique intensive et la façon dont celle-ci constitue un plan. Le plan de consistance "travaille ", se construit pied à pied et prépare la déterritorialisation sur fond d'une synthèse d'hétérogène qui s'articule selon trois temps : 1) créer un « milieu » (champ intensif), 2) produire des matières d'expression (territoire), 3) entraîner un mouvement excédentaire (déterritorialisation). Ces trois temps sont constitutifs des «systèmes intensifs » physiques, thermodynamiques, énergétiques, embryologiques, linguistiques etc., et des individuations spécifiques qu'ils produisent. Cet axe ternaire, essentiel dans la pensée de Deleuze et antérieurement même à sa rencontre avec Guattari, touche à l'expression, sous le double mouvement spatial et temporel de déterminations dynamiques. Bien que difficile à présenter brièvement, je voudrais résumer les traits principaux de cette conception des dynamismes qui me parait essentielle pour une approche intensive et transversale, et plus précisément celle d'une pensée-musique.

\section{L'esthétique intensive, un théâtre des dynamismes}

8 La question qui intéresse Deleuze est la captation de rapports matériaux-forces non pré-établis. Il est nécessaire, pour cela, de libérer la virtualité des variétés spatiales et temporelles de ce qui en préjuge dans l'Idée. Quelles déterminations se donne-t-on pour penser les rapports entre les dynamismes et l'énergie, la processualité des forces, la représentation des choses en général? Dès 1967, Deleuze introduit dans la Méthode de dramatisation ${ }^{4}$, dont le contenu sera repris par la suite dans Différence et répétition, un principe de différenciation, sous le double mouvement spatial et temporel de déterminations dynamiques. La différenciation ou actualisation de mouvements prend en compte, avec la plus grande exigence et les plus grandes précautions, la façon dont le champ de la représentation se « détermine ». La différenciation se joue au niveau de la formation de l'Idée, en tant qu'expérience, selon un constructivisme matériel immanent que l'on peut considérer comme le fondement des concepts ultérieurs de ritournelle et de déterritorialisation. Deleuze expose avec force le principe de la réciprocité des dynamismes sub-représentatifs, déterminant l'expérience intensive (individuation) selon un double mouvement complémentaire de qualification et d'organisation. Sous la déterritorialisation, la question de l'émergence de l'événement repose sur un champ protodynamique asignifiant et le jeu actif de déterminations spatiales et temporelles. L'expression et la capture de matériaux-forces sont au cœur de la problématique des déterminations de l'espace et du temps. Les dynamismes travaillent sous toutes les formes et les étendues qualifiées de la représentation. Bien qu'ils soient ordinairement recouverts par les étendues et les qualités constituées, « Il faut en 
faire le relevé dans tous les domaines ${ }^{5}$. Ces mouvements constituent les conditions de la représentation de tout objet ou état de chose, selon les qualités qu'il possède et l'étendue qu'il occupe, espèces et territoires.

En cet ancrage, le problème de l'expression est l'objet d'une recherche constante. La plupart des rencontres que Deleuze opère avec la littérature, la musique, le cinéma se situent sous la représentation, dans cette région « distincte et obscure » où se mène un combat pour libérer des matériaux-forces, affects et percepts. Deleuze s'attache à relever minutieusement (intensivement) ce qui a été tenté, l'objet et la modalité de la capture, pour donner un nom à cet événement: écrire, peindre... c'est une opération de différenciation, c'est "actualiser une Idée ». Son intérêt pour les conditions de l'émergence, les processus de trans-codage, les passages à la représentation, les modalités de la pensée, l'amènent à convoquer tous les champs intensifs susceptibles de rendre compte des aptitudes à configurer des relations de corps, de signes, de forces... Il ne s'agit en aucun cas d'application, mais plutôt de la saisie d'un diagramme intensif qui se détache par individuations.

Deleuze montre comment l'expérience de la différenciation trouve une individuation spécifique dans chaque domaine. Les dynamismes spatio-temporels propres à chaque milieu intensif acquièrent des spécifications et des modes d'extension particuliers selon le champ d'individuation. Cependant, tout système individuant - qu'il soit d'ordre physique, psychique, esthétique, politique -, répond à un ensemble de caractéristiques : les milieux d'individuation sont agités de différences d'intensités. Monde de bougements, de remuements encore sourds, aveugles, sans mémoire, pour des sujetsébauches non encore qualifiés ni composés, plutôt patients qu'agents ${ }^{6}$. Ces mouvements, que seul "l'embryon» peut supporter, sont expression, rapports intensité/vitesse, différences, avant la représentation et conditions de l'expérience. Les dynamismes spatio-temporels, en tant que vecteurs d'intensité, déterminent des directions de développement (embranchements, spécifications) et des phénomènes partitifs (plissements, étirements) qui répartissent les points remarquables et distribuent les singularités dans le champ intensif. Un champ intensif d'individuation se construira sur des séries de bordures hétérogènes ou disparates. La mise en communication des séries, sous l'action d'un différenciant (précurseur sombre), induit des phénomènes de couplage entre les séries, de résonance interne dans le système et de mouvement forcé sous forme d'une amplitude qui déborde les séries de base ellesmêmes ${ }^{7}$. La différenciation est actualisation, double mouvement de spécification et d'organisation. Qu'est-ce qui se joue? La qualification d'une espèce et l'organisation d'une étendue. Mouvements dont le tracé est morphogenèse, individuation d'un objet dans un champ spatial et temporel, selon ses deux aspects complémentaires: qualités et extension. Il est à noter que la question de la différenciation a, dans le domaine de la musique, partie liée avec un principe de « libre création » affirmé par Scriabine ${ }^{8}$. C'est aussi l'enjeu d'une position critique des relations sonores à l'œuvre chez certains musiciens, de Varèse à Messiaen, de Nono à Ligeti, ou plus récemment Horacio Vaggione ${ }^{9}$.

11 Deleuze souligne ensuite le caractère transitif, "en écho", des dynamismes subreprésentatifs qui constituent le matériau-force des champs expressifs ${ }^{10}$. Cette transitivité nous intéresse particulièrement pour comprendre comment Deleuze met en place un plan intensif (planomène) qui va ensuite permettre des rencontres hétérogènes sur le mode de dynamismes asignifiants susceptibles de s'actualiser et de 
circuler au niveau de racines prématérielles, entre la littérature, la peinture, la musique, le cinéma, mais tout aussi bien les sciences, la philosophie, le champ politique. L'esthétique intensive suppose que les modalités dynamiques et leurs déterminations dans la sensation, dans la pensée, dans la subjectivation psychique et politique, communiquent. Et la rencontre est à ce niveau, dans la mise en relation, bords à bords, de séries intensives hétérogènes et de captures réciproques. Étendre le concept philosophique aux matières esthétiques tend vers cette conviction que « quelque chose d'autre » peut être gagné, en deçà des formes pré-établies de la représentation. Il s'agit de donner la mesure du travail de l'expression dans toutes ses dimensions, d'ouvrir un plan nouveau pour l'exercice de la pensée. Une détermination intensive, spatiale et temporelle, traverse tous les arts et tous les champs de la pensée : pensée-plastique, pensée-cinéma, pensée-musique...

Cette présentation un peu aride des caractéristiques de la pensée différenciante de Deleuze, nous place donc au niveau des dynamismes et des déterminations dans la pensée. Deleuze analyse les divers systèmes d'individuation: esthétique, plastique, musical, littéraire, mais aussi scientifique, politique, éthologique dans lesquels les déterminations spatio-temporelles de la représentation s'éprouvent dans une production spécifique. Il relève les stratégies, les captures, les modes de connexion opérés par ces différents champs d'individuation. C'est donc au niveau de l'effectuation d'un diagramme intensif que les différents champs d'individuation esthétiques, politiques, philosophiques, scientifiques, communiquent, sous la représentation, au niveau des dynamismes ${ }^{11}$. Et c'est dans ce mouvement et dans cette région "distincteobscure » que Deleuze se tourne vers la musique, posant la question : quelle est la façon dont la musique affronte l'indifférencié, quel diagramme de déterminations spatiotemporelles met-elle en place pour libérer ses « matériaux-forces »?

\section{Bords à bords}

Liane Mozère nous fait remarquer, dans son appel à colloque, combien les notes de bas de pages sont nombreuses dans les écrits de Deleuze et Guattari, à quel point la diversité «rhizomatique» des branchements s'exerce dans la polyvocité et la polyphonie des références. Davantage que citées, ces voix parlent et renvoient à des mondes souvent connectés de façon inédite. Quel est le ressort de ce chantier invitant les arts, les sciences, l'esthétique, la politique à opérer des rencontres? L'enjeu des multiplicités spatiales et temporelles se développe avec une théorie «machinique » de l'expression qui se rapporte à l'intensif, présentdans chaque domaine de la pensée, chacun ayant sa façon de s'y confronter. Ni les images, ni la musique, ni la philosophie, ni les sciences ne peuvent répondre de mouvements intensifs, si ce n'est pour ellesmêmes. D'un côté, Deleuze et Guattari expriment la tâche de la philosophie : créer des concepts. Et de l'autre, celle de l'art: repousser les limites de la représentation. Les tâches sont distinctes, les spécificités exposées dans Qu'est-ce que la philosophie?, rappellent à chacune leur autonomie, leur direction propre. Les sciences procèdent par fonctions, la philosophie crée des concepts, l'art aura à faire aux affects et aux percepts. Il n'y a pas de confusion, à chaque champ d'individuation ses outils. Cependant l'expression et les productions machiniques se présentent sous un angle transversal et chevauchent les catégories. 


\section{L'agencement musical} représentation sont, comme nous l'avons vu, posées dès Différence et répétition au niveau de la différenciation et de l'individuation, la rencontre productive avec le modèle musical se constitue dans le courant des années 1970, dans la rencontre avec Félix Guattari. Le développement de la notion d'agencement machinique prend forme, dans L'Anti-CEdipe, avec la confirmation de la notion de consistance et une succession d'opérations partitives (distributions de la coupure sur le flux continu) ${ }^{12}$. Mille Plateaux développera l'approche d'une hétérogenèse sémiotique et fait appel en quelque sorte à un nomos, musical entre autres, dans une stratégie qui entend se démarquer du structuralisme: " "Comment la musique peut-elle nous aider à concevoir les productions d'un diagramme spatio-temporel ?", “Qu'est-ce qui n'est pas encore la musique, mais que seule la musique, dans sa spécificité, réussit à emporter ?" "Ces questions, souvent renouvelées, indiquent le champ technique et intensif appréhendé par la problématisation musicale, sous l'angle d'un agencement capable de produire de nouveaux types de réalité.

C'est donc sous ce double aspect, technique et intensif, que l'agencement musical et ses dispositifs sont interrogés. Traversée par une dimension diagrammatique, la musique procède en effet par spécification (analyse des propriétés, des composantes acoustiques), distribution des composantes (typologies de parentés, séries, modes, gammes) et stratifications partitives (accords, agrégats, polyphonie) ${ }^{13}$. Il y a effectuation d'un diagramme, avec une information connectable, déconnectable, transposable, assignable, disposée à la réorganisation de fonctions. La partition est un dispositif de coordonnées, avec son axe horizontal du temps, son axe vertical des données, ses distributions modulaires et son produit transversal. L'instrument de musique lui-même est un transducteur diagrammatique agençable selon ses définitions physiques, mécaniques, algorithmiques, numériques ${ }^{14}$. L'établi musical est un champ opératoire, un dispositif de pensée intégrant la géométrie, la topologie, les schèmes et les dynamismes spatio-temporels dont l'issue est produit de chevauchements de catégories corporelles, psychiques, abstraites, sensori-motrices.... Matériau vibratoire sur lequel des fonctions non préétablies effectuent des opérations partitives, la musique fait intervenir des lois de groupage et de répartition polyphonique qui engagent les déterminations spatiales et temporelles sous leur double aspect qualitatif et distributif. Sans doute la notion de consistance et la question de sa mise en œuvre entretient-elle un rapport privilégié avec ces couplages, résonances internes et transcodages, associés au processus de la déterritorialisation. Si le champ diagrammatique spatial et temporel de la musique est en communauté avec les opérations de différenciation (spécification, distribution, couplage, résonance interne, produit transversal des rapports), il est aussi lié à des regroupement de forces collectives et à leur cohérence. La complémentarité indissociable de l'aspect qualitatif et de l'aspect distributif (partitif) qui intéressent Deleuze et Guattari en tout domaine celui de l'agencement des coordonnées spatiales et temporelles nécessaires pour tout objet de la représentation -, trouve le site d'une mise "bords à bords " de séries hétérogènes et de variables dissymétriques dans le champ matériel transversal de la musique. 
16 À ce titre, la musique entretient un rapport spécifique avec le territoire et la façon dont il se constitue, selon un marquage dans lequel l'expressif est premier. On se souviendra de la façon dont la ritournelle agence indissociablement des marquages (cris, postures, intensités) et du territoire ${ }^{15}$. La musique, dans sa capacité à produire des matières d'expression par (re)groupement de forces, par (ré)organisation de fonctions (effectuation de l'agencement diagrammatique), travaille à même le chevauchement du sémiotique et du matériel. La production de matières d'expression s'accompagne de la production d'outils, de techniques ${ }^{16}$. Ce chevauchement déclenche quelque chose qui dépasse le territoire: une autonomisation de l'agencement, une création, une déterritorialisation.

Deleuze et Guattari opèrent en quelque sorte une capture du champ diagrammatique propre à la musique et le généralisent au niveau opératoire des dynamismes spatiotemporels. Ils extraient des aspects du «modèle » musical et les rapportent sur des champs qui lui sont a priori exogènes, bien qu'ayant toujours une composante diagrammatique commune. Le modèle des espaces lisses et des espaces striés, par exemple ${ }^{17}$, vaudra comme diagramme spatio-temporel applicable aux modèles technique, maritime, mathématique, physique, sociopolitique. Il participe, dans ses opérations de différenciation, de définition réciproques et de distribution, à la notion d'agencement de plans et de strates. Synthèse de disparates particulièrement apte à produire les effets d'une machine abstraite, le champ élargi du modèle musical devient, en quelque sorte, emblématique de l'agencement de déterritorialisation. Le nomos musical contracte et assimile la pensée moléculaire sous son aspect de transcodage. La façon dont les composantes musicales tiennent, par production transversale des coordonnées, son dispositif d'inscription polyphonique, de transpositions d'échelles et de proportions, conditionne le "vecteur spécialisé » de déterritorialisation. La musique comme flux de déterritorialisation, se cristallise dans Mille Plateaux, avec la notion de Ritournelle, dans son rapport privilégié avec la déterritorialisation et les devenirs.

\section{De la ritournelle}

Quelle est l'affaire de la musique, quel est le contenu indissociable de l'expression sonore? Guattari et Deleuze font de la musique le lieu privilégié d'un processus transversal de variation. Lieu d'échange tendu entre les forces territorialisantes de la ritournelle et la composition d'une ligne de variation proprement musicale. La ritournelle fait point de préhension, territoire, pli sécuritaire, avec le risque d'un retour mélancolique au natal, mais c'est essentiellement une ligne potentielle dont les points peuvent se redistribuer, se mettre en mouvement: distribution polyphonique, variations mélodiques, variations de timbres, de vitesses, de dynamiques, de densité d'orchestration... « La musique est l'opération active, créatrice, qui consiste à déterritorialiser la ritournelle ».

De ce plan d'intensités parcouru d'affects non subjectivés, de vitesses et de lenteurs, Deleuze et Guattari font le plan prématériel essentiel de la musique, indissociable d'un plan de vie ou d'un plan « naturant », productif de nouvelles dispositions. L'événement, ce qui est en train de s'éprouver, l'heure, la lumière, le lieu, se place comme immédiateté, composé perception-action que capte la ritournelle. La ritournelle devient la marque, un composé affect-percept indiciel. Ce sont des faits impersonnels qui s'individualisent et c'est en quelque sorte cette résonance impersonnelle, "un enfant crie », « les oiseaux tournoient dans le ciel », « on entend le bruit des bottes »... 
que la ritournelle capte et conserve dans un motif, sous l'expression d'une matière harmonique, d'une séquence rythmique.

La littérature, la peinture, le cinéma produisent aussi des matières d'expressions, mais de manière différente. Si la ritournelle saisit et territorialise, la musique elle, emporte (déplace) les bottes, le rire, les oiseaux, la ronde et l'heure. Elle les déterritorialise de façon très particulière: en composant et recomposant dans le temps des rapports dont les signes sont variables. C'est dans ce rapport pied à pied du contenu et de l'expression que la musique fait passer des forces «non-sonores »: forces qui n'étaient pas encore sonores, forces de la terre, du temps, affects guerrier, devenir-animal, devenir-femme, devenir-enfant, devenir-machine... L'enjeu de la musique serait cette puissance de transformation, de mutabilité des devenirs (quelque chose qui aurait à voir avec des métamorphoses) lié au travail de la ritournelle: "reste-t-elle territoriale et territorialisante, ou bien est-elle emportée dans un bloc mobile qui trace une transversale à travers toutes les coordonnées ? ${ }^{18}$.

21 Comment la ritournelle fait-elle territoire, comment passe-t-elle du non-musical à une variation musicale déterritorialisée ? Trois opérations caractérisent ce dispositif de la ritournelle, qui désormais n'appartient plus ni à la musique, ni à la philosophie, mais se constitue entre-deux, dispositif transversal qui fait tenir un espace «trans-catégoriel ».

I. Créer un milieu (infra-agencement de traits intensifs). Liée à ce qui fait demeure, sol, reconnaissance, la première opération de la ritournelle est de créer un milieu, seuil d'agencement qui conjure les forces anéantissantes du chaos. Il s'agit de fixer un point fragile qui fait centre. C'est la peur de l'enfant dans le noir, qui chantonne pour se rassurer, le cercle à tracer pour une tâche à remplir, une œuvre à faire.

II. Produire du territoire (intra-agencement des forces terrestres). Comme nous l'avons $\mathrm{vu}$, la ritournelle ne se rapporte pas au territoire au titre d'une évocation ou d'une représentation de celui-ci, mais elle en produit une dimension expressive, directement constitutive. Dans sa fonction territorialisante, la ritournelle opère une sélection, une différenciation sérielle: c'est la distribution spatiale d'un agencement, le passage à la territorialité avec la génération de matières expressives. C'est l'expressivité elle-même qui fait territoire. Ainsi le plan d'organisation développe les forces terrestres de l'intraagencement, libérant autant de matières d'expression que de marques territoriales.

III. Le mouvement de déterritorialisation (inter-agencement de la ritournelle). La troisième fonction qui s'exerce sur le territoire est une opération de transversalité: "La transversale est une composante qui prend sur soi le vecteur spécialisé de déterritorialisation ». Il s'agit d'un vecteur qui agit entre lesagencements territorialisés, stratifications, matières d'expression. Ce sont des composantes de passage qui établissent des relations non prélocalisées, sur les niveaux moléculaires inséparables de toutes les composantes matérielles des matières d'expression ${ }^{19}$. Le rapport essentiel se présente ici comme un rapport direct matériau-forces. Le matériau, c'est une matière molécularisée, qui doit à ce titre "capter» ses forces ${ }^{20}$. L'inter-agencement de la ritournelle déterritorialisée-déterritorialisante capte et intègre des composantes énergétiques, moléculaires, révèle des potentialités, déplie de nouvelles matières et de nouvelles transitions, «C'est un prisme, un cristal d'espace-temps qui agit sur ce qui l'entoure, son ou lumière, pour tirer des vibrations variées, des décompositions, projections et transformations... ${ }^{21}$. Dans ce mouvement transversal, surgissent de nouvelles populations et de nouvelles perceptions. 


\section{La pensée-musique}

En mettant en rapport les effectuations diagrammatiques propres à la musique avec les dynamismes prématériels et les agencements machiniques, Deleuze et Guattari situent la musique dans son extériorité constitutive, dans son rapport au monde. Ils soulignent le produit trans-catégoriel de ses individuations, celui d'un champ diagrammatique qui pratique une mise "bords à bords» de séries hétérogènes, et relève d'un constructivisme matériel transversal capable d'inventer des outils en prise avec un phylum machinique ${ }^{22}$. Ils désignent en même temps l'engagement d'une énonciation subjective qui ne concerne pas que la musique: celle d'une constante déterritorialisation du sujet, une constante épreuve $\mathrm{du}$ « milieu » en rapport avec les forces et les dynamismes. L'agencement musical serait caractérisé par le chevauchement du subjectif, de l'intensif et du diagrammatique. La pensée-musique, ancrée dans un rapport prématériel avec le territoire, aurait à voir avec l'établissement d'un rapport direct matériau/force et la captation de rapports force du temps/énergie.

Ce que nous lèguent Deleuze et Guattari serait davantage de l'ordre d'une posture, d'une réflexion envers la notion de dispositifs, d'agencements, d'outils de pensée aptes à aborder ces rapports matériaux-forces, plutôt que des outils finis. L'effectuation diagrammatique engage une pertinence à concevoir des outils en rapport avec un milieu, aptes à des configurations de variables, dont les fonctions peuvent se réorganiser. Plus que des systèmes binaires (disjonction exclusive), ce sont des matrices qui acceptent des signes hétérogènes, polyphoniques, qui opèrent des mutabilités, des connections et des reconnections. La production «machinique» concerne la mise en place des conditions sous lesquelles ces variables réalisent un couplage transversal, "bords à bords", entre des séries disparates et leurs composantes remarquables de passage ${ }^{23}$ ). Il n'y a pas de limite ni d'épuisement de connexion pour des objets de la représentation qui ne sont pas donnés, pour une expérience de la représentation qui se constitue comme champ d'individuation. En quoi ces outils polyvoques, "transcatégoriels", nous intéressent-ils aujourd'hui ? En quoi le chevauchement «transcatégoriel » d'une pensée-musique a-t-il à voir avec les champs imbriqués de la politique, de la perception et des agencements de vie? En quoi la Ritournelle et ses devenirs concerne-t-elle le champ du socius, le paysage vivant des affects, les formes du temps et de la vie dans laquelle nous nous mouvons?

\section{NOTES}

1. . Pascale Criton, « L'invitation », Deleuze épars. Approches et portraits, Hermann, 2005.

2. . «Deleuze avait une façon très personnelle de cadrer les propositions relevant de la musique. Les forces non-sonores de la musique, le temps non-chronologique... Ces expressions me semblaient paradoxales! Comment l'intemporel peut-il avoir une incidence réelle dans le sonore? Nous nous mettions d'accord sur des définitions à propos de l'acoustique, des gammes, des modes, du mineur et du majeur, des échelles, des intervalles, du continuum, de l'organisation 
harmonique et contrapunctique et nous revenions fréquemment sur ces foyers d'intérêt ayant trait au chromatisme dans leurs rapports à l'expression et aux formes. La notion de diagramme et de production transversale du modèle musical, celui des espaces lisses et des espaces striés en particulier (voir Pierre Boulez, Penser la musique aujourd'hui, Genève, Gonthier, 1964), fit l'objet de lectures et de réflexions. Nous eûmes de nombreuses réflexions sur les séries harmoniques, les partiels et composantes du son, en particulier au cours de l'étude de Leibniz, dont Deleuze recommandait fortement la lecture aux musiciens. D'autres fois, nous développions des recherches à propos des affects et de l'inflexion vocale dans la musique baroque, ou encore des signaux et comportements animaliers et des formes qui pouvaient en découler, de la caccia aux rondo et ritornelli. C'est ainsi que certaines notions sont devenues déterminantes dans Mille Plateaux », dans Pascale Criton, «L'invitation », op. cit., p.60.

3. Gilles Deleuze et Félix Guattari, Mille Plateaux, Paris, Minuit, 1980, p. 424.

4. . Gilles Deleuze, "La méthode de dramatisation", Bulletin de la Société française de Philosophie, 61e année, $n^{\circ} 3$, juillet-septembre 1967, rééd. dans Gilles Deleuze, L'île déserte et autres textes (textes et entretiens 1953-1974), David Lapoujade, Minuit, 2002, p. 131-144.

5. . Gilles Deleuze, Différence et répétition, op. cit., p. 276.

6. . Ibid., p. 276-285.

7. . « Le dynamisme comprend alors sa propre puissance de déterminer l'espace et le temps, puisqu'il incarne immédiatement les rapports différentiels, les singularités et les progressivités immanentes à l'Idée. ", Gilles Deleuze, Différence et répétition, op. cit., p. 282. Et Gilles Deleuze, « La méthode de dramatisation », L’île déserte et autres textes, op. cit., p. 136.

8. . Pascale Criton, « Le cerveau transfini », Chimères n 27 , Paris, 1996, p. 55-66.

9. . Pascale Criton, «Mutation et processualité dans la pensée musicale de Horacio Vaggione », Horacio Vaggione-Espaces composables, Paris, L'Harmattan, 2007.

10. . Gilles Deleuze, Différence et répétition, op. cit., p. 283.

11. . Pascale Criton, "L'esthétique intensive ou le théâtre des dynamismes ", Deleuze et les écrivains. Littérature et philosophie, sous la direction de Hervé Micolet et Bruno Gelas, Éd. Cécile Defaut (PUF), Nantes, 2007.

12. . Félix Guattari, Écrits pour l'Anti-@Edipe, textes agencés par Stéphane Nadaud, Éditions Lignes et Manifestes, 2004.

13. . Pascale Criton, "Continuum sonore et schèmes de structuration », Musique, rationalité, langage. L'harmonie : du monde au matériau, «Cahiers de philosophie du langage », $n^{\circ} 3$, Paris, L'Harmattan, 1998, p. 73-88.

14. . Pascale Criton, "Tekhné et expressivité», Musiques, arts et technologies. Pour une approche critique, Paris, l'Harmattan, coll. « Musique-Philosophie », 2004.

15. . Pascale Criton, «À propos d'un cours du 20 mars 1984. La ritournelle et le galop », dans Gilles Deleuze, une vie philosophique, sous la direction de Éric Alliez, Institut Synthélabo (PUF), 1998, p. 513-523.

16. . Car s'il « n'y a d'histoire que de la perception, [...] il n'y a d'imagination que dans la technique ", "L'artisan cosmique et le phylum machinique », Gilles Deleuze et Félix Guattari, Mille Plateaux, p. 414, et la relation affects-percepts, ibid., p. 428.

17. . Pierre Boulez, Penser la musique aujourd'hui, Genève, Gonthier, 1964, p. 93-113.

18. . Gilles Deleuze et Félix Guattari, Mille plateaux, op. cit., p. 415.

19. Sur le niveau moléculaire des matières d'expression et les transversales de déstratification, Gilles Deleuze et Félix Guattari, Mille plateaux, op. cit., p. 412-416.

20. . Ibid., p. 422.

21. . Ibid., p. 430.

22. . «La création d'une ligne transversale repose sur le double mouvement d'un plan abstrait prématériel, ou plan de consistance d'une part, et d'un plan d'élaboration logique ou plan d'organisation, d'autre part. Les deux plans sont indissociables, liés dans un mouvement de 
production réciproque, ils ne cessent de s'échanger ", "Souvenirs d'un planificateur ", Gilles Deleuze et Félix Guattari, Mille plateaux, op. cit., p. 325-333, p. 363-367 et suivantes.

23. . «La déterritorialisation implique la coexistence d'une variable majeure et d'une variable mineure qui deviennent en même temps : la déterritorialisation est toujours double... Les deux termes ne s'échangent pas moins que l'autre ", dans Gilles Deleuze et Félix Guattari, Mille Plateaux, op. cit., p. 377.

\section{RÉSUMÉS}

Au cours des années 1970, époque marquée par le structuralisme, Deleuze et Guattari se tournent vers la question d'un rapport nouveau entre l'expression et les dispositifs de la pensée. Leur point de vue s'établit avec le projet d'une hétérogenèse sémiotique transversale et prend appui sur la recherche d'outils de pensée puisés dans tous les domaines de la représentation. Qu'est-ce qui traverse les champs expressifs, du politique au technique, du social au psychique? La linguistique, l'ethnologie, l'éthologie, la psychiatrie, les mathématiques, non moins que l'esthétique, la littérature, la peinture, le cinéma sont convoqués. C'est dans cette perspective que le domaine particulier de la musique est interrogé. La musique peut-elle être sollicitée pour une pensée transversale? Quels sont ses outils, quel est son champ opératoire? En quoi la musique peut-elle nous aider à penser?

Cross-edges: towards music-thought During the seventies, characterised by structuralism, Deleuze and Guattari look towards a new relation between expression and frame of thinking. They establish their point of view with a transversal heterogeneous semiotic project and rely on tools drawn from every fields of representation. What is running through expressive domains, from political to technical, from social to psychical? Linguistics, ethnology, ethology, psychiatry, mathematics as well as aesthetics, literature, painting or movie are invited. In the same prospect, the particular field of music is called up. Can music be requested for a transversal thought? What are its operative modes and tools? In what way could music help us in thinking?

Von Bord zu Bord Folgende Frage wird hier gestellt: kann die Musik zu einem transversalen Denken zugezogen werden? Was sind ihre Arbeitsinstrumente? Welch ist ihr Wirkungsbereich (Operationsfeld)? Inwiefern kann uns die Musik beim Denken behilflich sein?

\section{AUTEUR}

\section{PASCALE CRITON}

Pascale Criton est compositrice et docteur en musicologie du xxe siècle. Elle explore la variabilité du son au niveau des techniques de jeux instrumentaux, d'accords et de la synthèse numérique. Elle est actuellement compositeur en recherche à l'Ircam. Elle rencontre Gilles Deleuze en 1975 et sera son interlocutrice durant de nombreuses années. Son catalogue d'œuvres, édité chez Jobert, est consultable sur www.pascalecriton.org. Un recueil d'entretiens et d'articles consacré à son travail est paru dans la collection à la ligne : Pascale Criton, Les univers microtempérés (2e2m 1999), ainsi qu'un Cd monographique chez Assaï (2003). 